\title{
OBTENTION OF PORCINE AMINOPEPTIDASE-N TRANSGENIC MICE AND ANALYSIS OF THEIR SUSCEPTIBILITY TO TRANSMISSIBLE GASTROENTERITIS VIRUS
}

\author{
L. Benbacer, M.-G. Stinackre, H. Laude, and B. Delmas \\ Unité de Virologie et Immunologie Moléculaires \\ Institut National de la Recherche Agronomique \\ F-78350 Jouy-en-Josas, France
}

\section{ABSTRACT}

To obtain a laboratory animal model for transmissible gastroenteritis virus (TGEV) infection, transgenic mice $(\mathrm{Tg})$ were produced by introducing two porcine aminopeptidase-n (APN) cDNA-derived constructs into the mouse genome. In the first construct, the APN cDNA was fused in 5' with the $1 \mathrm{~kb}$ upstream region of the APN gene and in 3' with the SV40 small intron and polyadenylation site. In the second construct, the 5' end of the APN cDNA was replaced by the corresponding domain of the APN gene comprising the three first introns, an additional intron (the rabbit $\beta$-like globine intron 2) was inserted at the 3' extremity of the construct and the resulting DNA stretch was placed under the control of the rat intestinal fatty acid-binding protein (I-FABP) gene promoter. Transgenes were obtained with these two constructs, and RNA expression was evidenced by RT-PCR with the second construct in a transgene lineage. Using two different immunoassays, expression of the porcine APN protein was not detected in the transgenic intestines of animals of the RT-PCR positive lineage. Northern blot analyses did not revealed TGEV replication in infected adult mice. Additional assays will be carried out on young animals to detect potential TGEV susceptibility.

\section{INTRODUCTION}

TGEV enters the target cells by binding to a specific surface receptor, porcine APN, (Delmas et al., 1992), followed by the fusion between viral and cellular membranes to re- 
lease the genomic RNA into the cytoplasm. In susceptible animals, TGEV infection starts with ingestion of the virus followed by a primary infection of enterocytes of the jejunum and, to a lesser extent, of the duodenum and the ileon (reviewed by Enjuanes and Van der Zeijst, 1995). TGEV also replicates with a low efficiency in the upper respiratory and in alveolar macrophages. In contrast, porcine respiratory coronavirus, a natural variant of TGEV which also uses APN as a receptor, replicates efficiently in the respiratory tract, but poorly in the gut. The mechanism for this host cell restriction is not known. In addition, TGEV and PRCV replication is mainly restricted to these cell types and systemic infection were never clearly evidenced (reviewed by Laude et al., 1993). APN is present in almost all tissues, is highly expressed in kidney proximal tubule cells and in enterocytes but also, in less amount, in other epithelial cells (reviewed by Norén et al., 1997).

The pig species is the only natural host for TGEV. Some other species such as canine and feline species naturally infectable by antigenically-related viruses have been experimentally infected by TGEV, but infections were found to be asymptomatic (reviewed by Holmes and Compton, 1995). Identification of APN as a cellular receptor for TGEV has opened new perspectives in developing transgenic laboratory animal models to study TGEV pathogenesis. To this purpose, we have constructed two porcine APN cDNA-derived minigenes to target expression in epithelial cells of the gut and of the respiratory tract. Transgenic mice obtained with the two minigenes were analyzed for their susceptibility to TGEV infection.

\section{METHODS AND MATERIALS}

\subsection{Plasmids Construction}

For minigene 1, the 2.8-kb EcoRI/EcoRI fragment of porcine APN cDNA was inserted downstream of the porcine APN gene DNA fragment HindIII/EcoRI made of 1.2-kb upstream of the transcription initiation site and part of the exon I. The polyadenylation signal and intron sequences of SV40 small T antigen were amplified by PCR using primers that create a BglII restriction enzyme site at the 5' end and a Xbal restriction enzyme site at the 3' end. This PCR product was then cloned at the 3' end of the construct. For minigene 2, the $3.3-\mathrm{kb}$ BamHI/XhoI fragment of the cDNA encoding porcine APN was cloned downstream of the rat I-FABP promoter. APN intron I (provided by J. Olsen, Copenhagen) was inserted by using EcoRI and AccI enzyme restriction sites present in the cDNA. Introns 2 and 3 were amplified by PCR with genomic porcine DNA as template using primers with AccI and BspE1 restriction enzyme sites present in the cDNA. The PCR product was cloned in the relevant restriction sites of the construct. A 2.2-kb BamHI/BspEI DNA fragment containing the rabbit $\beta$-globin 3' intron and polyadenylation signal was inserted downstream of the APN coding region. The structure of the final plasmids was confirmed by restriction enzyme digestion. For microinjection, minigene 1 (5.3$\mathrm{kb})$ was cleaved by XhoI and XbaI, minigene $2(7.5-\mathrm{kb})$ by NotI and XhoI to remove vector sequences. The inserts were purified by agarose gel electrophoresis and diluted before microinjection.

\subsection{Microinjection and Production of Transgenic Mice}

DNA inserts were microinjected in fertilized eggs of the F1 generation of C57Bl6 $\mathrm{x}$ CBA mice and transgenic mouse foundators were identified by PCR assay of tail DNA, 
using adequate primers. Southern blot analysis was also performed to evaluate the number of copies per genome.

\subsection{DNA and RNA Isolation}

Tails of newborn or 3-week-old mice were cut and digested in $300 \mathrm{mM}$ sodium acetate ( $\mathrm{pH} 8$ ), $1 \mathrm{mM}$ EDTA, $1 \%$ sodium dodecyl sulfate, and $150 \mu \mathrm{g}$ of proteinase $\mathrm{K}$ per 750 $\mu l$. Undigested debris were removed by centrifugation. After two phenolic extractions, DNA was precipitated with isopropanol and dissolved in distilled water. Total RNA was isolated from small intestine by homogenization in $4 \mathrm{M}$ guanidium thiocyanate with a homogenizer followed by acid phenol extraction. RNA was precipitated with isopropanol and dissolved in distilled water.

\section{RESULTS}

\subsection{Minigene 1}

Transgenic mice were generated by using a construct in which porcine APN cDNA was linked to the porcine APN promoter region containing $1.2 \mathrm{~kb}$ of upstream sequence and, in the 3' part of the minigene, the intron and polyadelylation signal of SV40 $t$ antigen (Fig. 1). This construct, named minigene 1, was expected to drive APN expression in epithelial cells. To ensure that minigene 1 will produce a functional TGEV receptor, plasmid DNA was transiently transformed into non-permissive MDCK cells followed by TGEV infection. Neosynthesized viral antigens were revealed in cells transformed by minigene 1 plasmid, but not in cells transformed with the vector alone (not shown). The minigene 1 was excised and microinjected into fertilized mouse eggs. Transgenic mice were screened for the presence of the transgene by PCR and Southern blotting analysis of tail DNA (Fig. 2). Four transgenic founders were identified, and two of them (founders 2 and 11 ) were used to further study.

Northern blot hybridization analysis was used to determine whether porcine APN RNA is expressed in the small intestine and in the liver of minigene 1 transgenic offspring

A. Minigene 1

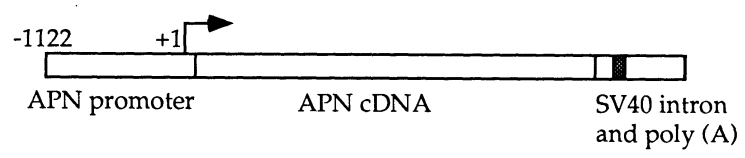

B. Minigene 2

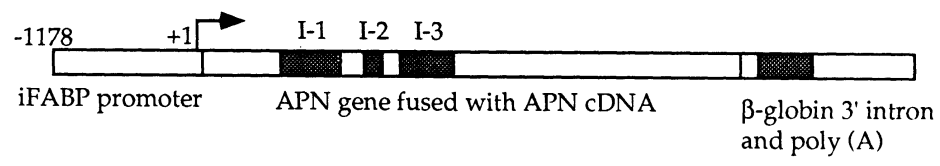

$1 \mathrm{~kb}$

Figure 1. Structure of the minigenes used to generate transgenic mice. The introns are indicated in grey. I-1 to -3 are the first 5 ' introns of the porcine APN gene. Arrows indicate the site of initiation of transcription. 
A

$\begin{array}{llllllllllll}1 & 2 & 3 & 4 & 5 & 6 & 7 & 8 & 9 & 1011 & 12\end{array}$

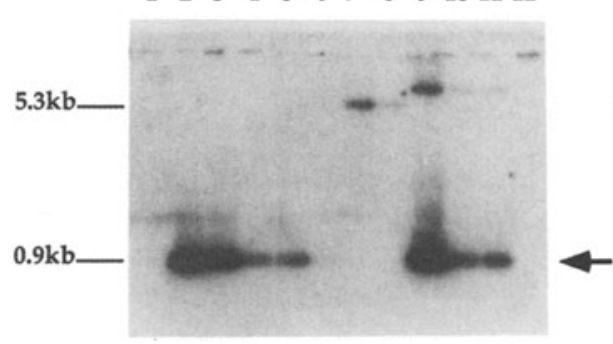

B

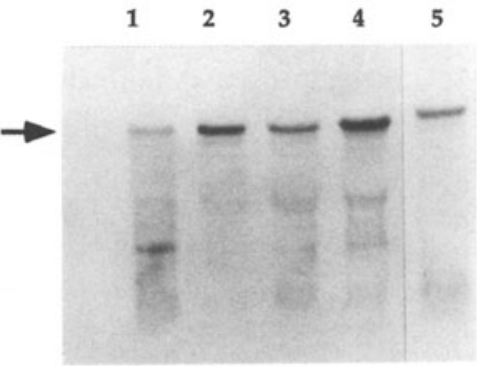

Figure 2. (A) Southern blot analysis of transgenic founders for minigene 1. Tail DNA of normal (lane 1), $\mathrm{Tg} 2$ (lane 2), $\operatorname{Tg} 11$ (lane 3), $\mathrm{Tg} 13$ (lane 4), $\mathrm{Tg} 24$ (lane 5) and non- $\mathrm{Tg}$ mice (lanes 6, 9, 10, 11 and 12) was digested by Ncol enzyme. All these lanes contained equal amounts of DNA, as judged by ethidium bromide staining. Lanes 7 and 8 contain the equivalent of 10 and 2 copies/genome of minigene 1 cut Xhol-Xbal (5.3-kb), respectively. In lanes $9,10,11$ and 12, the equivalent of 20,10,5 and 0 copies/genome were added bebore DNA digestion. The DNA probe was the $0.9-\mathrm{kb}$ Ncol-Ncol fragment of the cDNA. The arrow indicates the expected position of the porcine APN DNA of transgenic mice. (B) Northern blot analysis of total cell RNA from the swine ST cell line (lane 1) and from the small intestine of a normal mouse (lane 2), of the Tg2 offspring 2.15 (lane 3), of pig (lane 4) and of the $\mathrm{Tg} 11$ offspring 11.09 (lane 5). The arrow indicates the expected position of the endogenous swine or mouse APN mRNA.

of founders 2 and 11. Analyses carried out with probes deriving from the porcine APN RNA revealed the 3.3-kb endogeneous mRNA encoding for the murine APN, but a $3.5-\mathrm{kb}$ band consistent with that predicted from the structure of minigene 1 was never detected (Fig. 2). In the same way, a $3.5-\mathrm{kb}$ band was not revealed by using as a probe the DNA stretch deriving from the SV40 DNA stretch present in the construct (not shown).

However, since a low concentration of receptor at the cell surface of target cell, at least in vitro, was found to be sufficient to confer susceptibility in cell culture (Delmas et al., 1995), infection assays were performed. Adult and new-born mice of the lineage 2 were orally infected with around $10^{6}$ PFU of TGEV to analyse their permissivity. No enteritis was observed up to 3 days after infection. Viral replication was assayed by Northern blot analysis, by indirect immunofluorescence experiments and by quantification of interferon induction. No TGEV replication could be evidenced by these different assays (not shown).

\subsection{Minigene 2}

We designed another minigene by using a promoter which was previously validated in transgenic animals to direct expression in enterocytes and by adding introns in the cDNA for efficient mRNA maturation. To direct expression of porcine APN to the enterocytes of transgenic mice, the promoter for rat I-FABP was chosen. The I-FABP gene product is expressed in differentiated, villus-associated, small intestine epithelial cells (Sweetser et al, 1987). This promoter was validated to direct expression of diverse proteins in the enterocytes of transgenic animals (Sweetser et al., 1988, Zhang and Racaniello, 1997). Genomic sequences for porcine APN introns 1-3 were included in the full-length porcine APN cDNA which was cloned downstream of the I-FABP promoter. In addition, a fragment containing the rabbit $\beta$-globin intron 2 and poly(A) polyadenylation 
signal was placed immediately downstream of the APN cDNA to ensure posttransciptional maturation and mRNA stability. To ensure that this construct, named minigene 2, produces a functional TGEV receptor, plasmid DNA was transiently transformed into receptor-negative BHK cells followed by TGEV infection. Neosynthesized viral antigens were revealed in cells transformed by minigene 2 plasmid, but not in cells transformed with the vector alone (not shown). Transgenic mice were produced with minigene 2 and screened for the presence of the transgene by PCR and Southern blotting analyses of tail DNA (not shown). Five transgenic founders, numbered 56, 58, 68, 116 and 126, were identified and used to further study.

Northern blot and RT-PCR analyses were carried out to determine whether porcine APN mRNA is expressed in transgenic animals of the lineages 56, 58, 68 and 126 . Transgenic RNA was only detected in the small intestine of offspring of the founder mouse 126 by reverse transcription-polymerase chain reaction (RT-PCR) with primers specific of the APN cDNA and of the $\beta$-globin exon 3 (Fig. 3). Cloning and sequencing of the 480 bases pair RT-PCR product revealed a correct fusion between the two DNA domains and the expected splicing of $\beta$-globin intron 2.

To determine if offspring of founder 126 was susceptible to TGEV infection, $2 \times 10^{6}$ PFU were inoculated into the jejunum of five two month-old mice of this lineage. No enteritis was observed up to 3 days after infection. Northern blot analysis of intestine RNA extracted 8,24 and $48 \mathrm{~h}$ post infection were used to vizualize TGEV replication. No viral subgenomic mRNA were detected in the mice (Fig. 4).

\section{DISCUSSION}

A susceptible mouse model for TGEV infection would have obvious advantages over the use of the porcine species, its natural host. This laboratory model could be easily used to study the pathology and the intestinal immune response associated to the infection and would facilitate the development of antiviral approaches. For this purpose, we constructed two different minigenes to obtain transgenic mice expressing porcine APN in en-

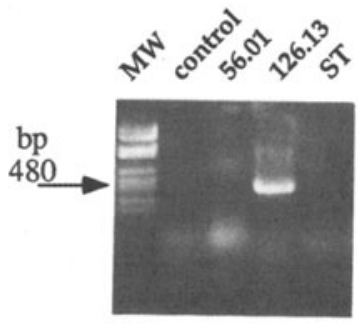

Primers: APN/Globin

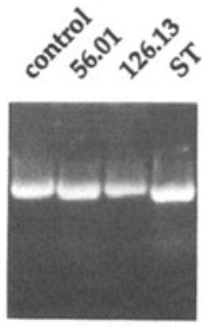

Actin

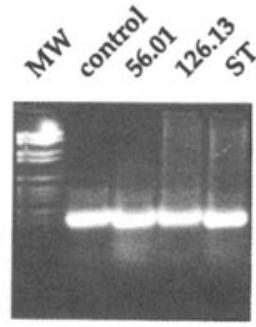

APN

Figure 3. RT-PCR analysis of transgenic minigene 2 mRNA in the intestine of mice from transgenic lines 126 and 56. Reverse transcription was done on total tissue RNA with an oligo(dT) primer. Beta-actin and murine APN cDNAs were used as positive controls. PCR of the APN/globin fragment was done with primers 5'TAATAGTGCCTGGTCCTTCCCGCCACCTGG and 5'-ATGAGACAGCACAACAACCAGCACGTTGCC. MW, molecular weight marker VI (Boehringer), control shows a negative control with a normal mouse and ST the PCR amplification obtained with the ST porcine cell line. Gels were stained with ethidium bromide. 


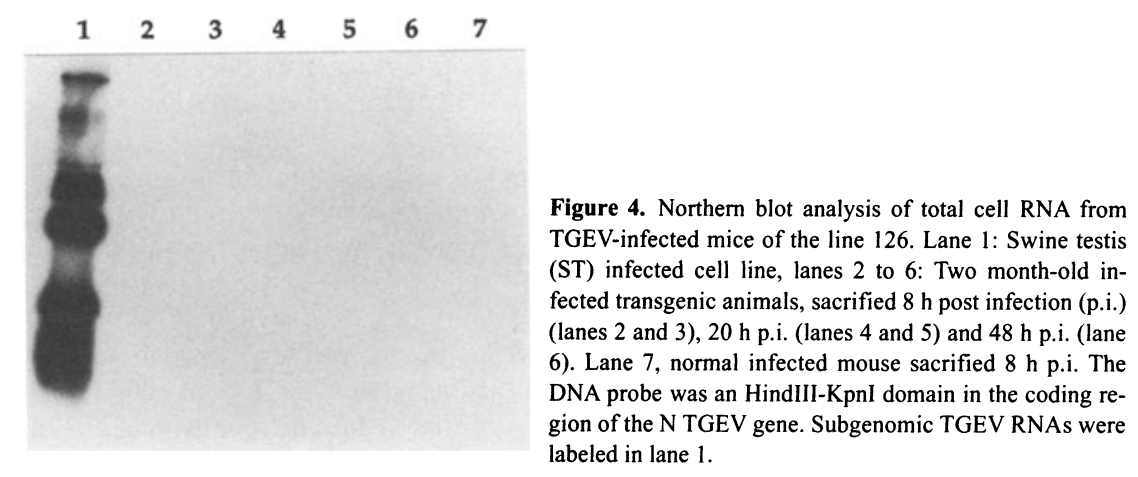

terocytes of the small intestine. In minigene 1 , the APN cDNA was fused with the $1 \mathrm{~kb}$ upstream region of the APN gene. We did not detect a transcriptional activity in the offsprings of two transgenic founders. This result could be due to absence of transcription, a defect of mRNA maturation or to the low number of lineages we analyzed. In minigene 2, the I-FABP promoter was found to be functional in one out the four lineages studied. A transcript was evidenced by RT-PCR in the lineage 126, but adult mice of this lineage were found not susceptible to TGEV infection as determined by Northern blot analysis.

There are several possible explanations for the failure of TGEV to replicate in the intestine of mice of the lineage 126. First, we were unable to detect the porcine APN in the transgenic animals by immunostaining of intestine sections (not shown) whereas in the mammal intestine, APN represent 5 to $8 \%$ of the total proteins present at the enterocyte brush border membranes. The concentration of receptor on the cell surface is possibly a critical factor for virus entry in the digestive tract. Secondly, mouse enterocytes possibly lack a functional host factor for TGEV entry or replication. Such a hypothesis has been recently proposed for the unability of poliovirus to replicate in enterocytes of transgenic mice expressing the poliovirus receptor (Zhang and Racaniello, 1997). In the same way, transgenic mice ubiquitously expressing human CD46, the receptor for measles virus, were not susceptible to virus infection (Horvat et al., 1996). Thirdly, we only infected adult mice and it would be of interest to infect newborns which are expected to be more susceptible than adults, as it is observed following experimental and natural infection of the swine.

The recent finding that feline APN can act as a functional receptor for TGEV in cell culture (Tresnan et al., 1996) raises the question of the capacity of TGEV to adapt to other mammals species. Indeed, TGEV was never reported to cause enteritis and to multiply efficiently in the cats. Thus, host factors other that the fixation of TGEV on APN may govern the spread of the virus and its ability to cause disease.

\section{REFERENCES}

Delmas, B., Gelfi, J., L'Haridon, R., Vogel, L.K., Sjöström, H., Norén, O., and Laude, H., 1992, Aminopeptidase $\mathrm{N}$ is a major receptor for the enteropathogenic coronavirus TGEV, Nature. 357: 417-419.

Delmas, B., Kut, E., Gelfi, J., and Laude, H., 1995, Overexpression of TGEV cell receptor impairs the production of virus particles, in: Corona- and related viruses, Volume 380 (P. Talbot, and G. Levy, eds.), Plenum Press, New York, pp. 379-385. 
Enjuanes, L., Van der Zeijst, B.A.M., 1995, Molecular basis of transmissible gastroenteritis virus epidemiology, in: The Coronaviridae (S.G. Siddell, ed.), Plenum Press, New York and London, pp.337-376.

Holmes, K.V., and Compton, S.R., 1995, Coronavirus receptors, in: The Coronaviridae (S.G. Siddell, ed.), Plenum Press, New York and London, pp. 55-71.

Horvat, B., Rivailler, P., Varior-Krishnan, G., Cardoso, A., Gerlier, D., and Rabourdin-Combe, C., 1996, Transgenic mice expressing human measles virus (MV) receptor CD46 provide cells exhibiting different permissivities to MV infection, J. Virol. 70: 6673-6681.

Laude, H., Vanreeth, K., and Pensaert, M., 1993, Porcine respiratory coronavirus-Molecular features and virus host interactions, Vet. Res. 24: 125-150.

Norén, O., Sjöström, H., and Olsen, J., 1997, Aminopeptidase N, in: Cell-surface peptidases in health and disease (A.J. Kenny, and C.M. Boustead, eds.), BIOS Scientific Publishers, pp. 175-191.

Sweetser, D.A., Birkenmeier, E.H., Klisak, I.J., Zollman, S., Sparkes, R.S., Mohandas, T., Lusis, A.J., and Gordon, J.I., 1987, The human and rodent intestinal fatty acid binding protein gene, J. Biol. Chem. 262: 16060-16071.

Sweetser, D.A., Hauft, S.M., Hoppe, P.C., Birkenmeier, E.H., and Gordon, J.I., 1988, Transgenic mice containing intestinal fatty acid-binding protein-human growth hormone fusion genes exhibit correct regional and cellspecific expression of the reporter gene in their small intestine, Proc. Natl. Acad. Sci. USA 85: 9611-9615.

Tresnan, D.B., Levis, R., and Holmes, K.V., 1996, Feline aminopeptidase N serves as a receptor for feline, canine, porcine, and human coronaviruses in serogroup I, J. Virol. 70: 8669-8674.

Zhang, S., and Racaniello, V.R., 1997, Expression of the poliovirus receptor in intestinal epithelial cells is not sufficient to permit poliovirus replication in the mouse gut, J. Virol. 71: 4915-4920. 